\title{
SPATIAL ASPECTS OF CENSUS DISTRICTING
}

\author{
S. Dezyani* and F. Karimipour \\ Department of Surveying and Geomatics Engineering, College of Engineering, University of Tehran, Iran-(sdezyani, \\ fkarimipr)@ut.ac.ir
}

KEY WORDS: Urban districting, Spatial partitioning, Census districting, Workload, Compactness

\begin{abstract}
:
Urban districting refers to partitioning of an urban area into smaller regions for a specific application in order to effectively facilitate and enhance the quality of municipal services. Among other considerations, which are imposed by the general problem or the application in hand, several factors in urban districting have spatial aspects, many of which have been disregarded in most of districting plans, and only descriptive measures have been considered. This paper explores the impact of spatial aspects on census districting, as an important urban districting. It proposes an approach that not only considers the workload, as the most effective criterion in census districting, but spatial criteria such as compactness, barriers and travers length are also involved. The implementation results of the proposed approach for a case study have been evaluated and discussed.
\end{abstract}

\section{INTRODUCTION}

Urban districting refers to partitioning of an urban area into smaller regions for a specific application. It is an important issue in planning, especially for large cities and metropolises, in order to facilitate and enhance the quality of municipal services provided by governmental organizations. Several factors are involved in urban districting, which can be classified into general constraints (e.g., urban size, population density and distribution), and application-dependent criteria (e.g., compactness and accessibility).

Many of the criteria used in urban districting problem have spatial aspects. Shape and geometry of districts, and their topological relationships are examples of such spatial criteria. Therefore, explicit deployment of their impact on the problem may improve the efficiency of existing districting systems. A variety of applications of urban districting have been the subject of many studies, including political districting (Alt et al., 1996; Bozkaya et al., 2003; Garfinkel and Nemhauser, 1970; Helbig et al., 1972; Hess et al., 1965; Mehrotra, 1998; Ricca et al., 2008; Ricca and Simeone, 2008; Yamada, 2009), census districting (Martin, 1998; Openshaw, 1995; Ralphs et al., 2009) and commercial districting (Salazar-Aguilar et al., 2010; SalazarAguilar et al., 2011).

Census districting, the case study of this paper, is an urban districting whose goal is providing a fair distribution of the workload to census officers. Although the most important criterion in census districting is the total workload of the blocks located in a district, it is influenced by spatial and geometrical criteria. This paper studies the spatial aspects of census districting problem and proposes an approach to explicitly involve them in the districting process.

The rest of the paper is structured as follows: Section 2 provides a review on spatial partitioning problem and its related concepts. In section 3, spatial aspects of census districting are introduced and quantified, which are evaluated through a case study implemented in Section 4. Finally, Section 5 concludes the paper and proposes ideas for future work.

\section{SPATIAL PARTITIONING}

\subsection{Problem Description}

In general, spatial partitioning problem can be described as decomposing of a large space into smaller subspaces subject to some desired criteria in order to ease of monitoring, planning and management (Salazar-Aguilar et al., 2011). If the original space is composed of a large number of basic spatial units (BSU), then spatial partitioning is aggregating or grouping of these units into larger zones (Figure 1) (Bozkaya et al., 2003; Macmillan, 2001; Salazar-Aguilar et al., 2010; Shirabe, 2005; Yamada, 2009). Spatial partitioning is a multi-objective optimization problem, for which several methods have been proposed based on heuristic and meta-heuristic algorithms (Bação et al., 2005; Bozkaya et al., 2003; Mehrotra, 1998; Wei, 2004) as well as the data structures used in computational geometry (Moreno-Regidor et al., 2012; Mu, 2004; Novaes et al., 2009; Ricca et al., 2008; Salazar-Aguilar et al., 2010; Svec et al., 2007; Torpelund-Bruin and Lee, 2009; Yamada, 2009).

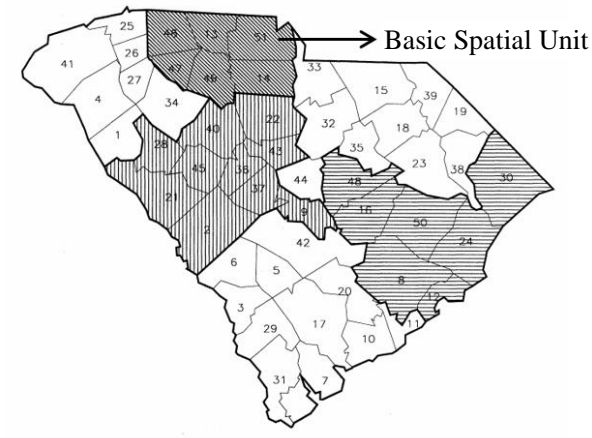

Figure 1. Spatial partitioning as aggregating or grouping of a large number of basic spatial units

Urban districting is a class of spatial partitioning problems that decompose an urban area into smaller regions for a specific application. The factors that are involved in urban districting 
can be classified into general constraints and applicationdependent criteria.

\subsection{General Constraints}

These constraints are independent of the purpose of partitioning and will be treated as hard constraints. Let $I=1, \ldots, n$ be the set of all basic spatial units and $J=1, \ldots, k$ be the set of all partitions, where $n$ and $k$ are respectively the total number of basic units and partitions.

2.2.1 Integrity: Integrity means that each basic spatial unit belongs to one and only one division (Bozkaya et al., 2003). Equation (1) guarantees the integrity condition:

$$
\sum_{j \in J} x_{i j}=1
$$

If the $i^{\text {th }}$ basic spatial unit is assigned to the $j^{\text {th }}$ division, then $x_{i j}=1$; otherwise $x_{i j}=0$.

2.2.2 Number of Divisions: In some applications, the number of divisions is determined prior to space partitioning. Equation (2) guarantees that the number of final divisions is the pre-determined value $k$ (Bozkaya et al., 2003; Ricca et al., 2011):

$$
\sum_{j \in J} x_{j j}=k
$$

2.2.3 Contiguity: In a contiguous area one can walk from any point to any other point without ever leaving the area. In other words, a contiguous area cannot have any holes (Figure 2).

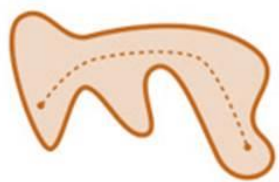

(a)

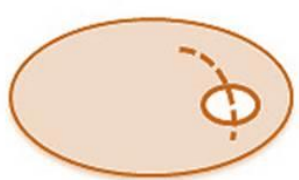

(b)
Figure 2. A (a) contiguous and (b) non-contiguous shapes

Several methods have been proposed to apply contiguity constraint in the partitioning problem, including contiguity matrix (Openshaw, 1977) and mixed integer programming (MIP) models (Cova and Church, 2000; Ricca et al., 2011; Shirabe, 2009; Zoltners and Sinha, 1983).

2.2.4 Non-empty Divisions: This constraint prevents creating empty divisions and ensures that each division contains at least one BSU.

2.2.5 Equality: This constraint guarantees that the difference of a set of parameters among the divisions is less than a certain threshold. i.e., they are as equal as possible, in order to achieve the maximum heterogeneity. For the parameter $P$, the objective function to be minimized is as follow:

$$
f(x)=\frac{\sum_{k \in K} \max \left\{P_{k}(x)-(1+\beta) \bar{P},(1-\beta) \bar{P}-P_{k}(x), 0\right\}}{\bar{P}}
$$

where $K$ is the number of divisions and $\bar{P}$ is the average of the parameter $P$ for all of the divisions.

\subsection{Application-dependent criteria}

These criteria will vary from one application to another. Most of the commonly used application-dependent criteria are (Bozkaya et al., 2003):

- Socio-economic homogeneity, which is considered in most of the political districtings.

- Compactness (similarity of the shape of the divisions to circles)

- Geometrical homogeneity (minimizing the variance of geometric characteristics such as areas, perimeters, etc.)

- Spatial interaction between divisions

- Accessibility to a certain location or object

- Avoiding natural or artificial barriers such as rivers or highways

- Similarity to other existing administrative districts, which provides shared use and results in ease of management.

Many of the above application-dependent criteria have spatial aspects. Therefore, explicit deployment of their impact on the problem may improve the efficiency of existing districting systems. However, many of districting plans disregard these criteria or at most consider them as second-order factors compare to the descriptive measures.

In the next two sections, we show how explicit involvement of the spatial aspects in census districting, as the case study of the paper, can provide more reliable results.

\section{CASE STUDY: CENSUS DISTRICTING}

Consider a set of urban population blocks with known workloads. The workload of a block depends on the number of households live in that block, and is the number of days takes for a team to census that block. The census districting partitions a set of such blocks into groups with a pre-defined total workload.

Most of the urban districts are once created and will remain unchanged for a long time or even forever; whereas, census districts must be updated before every censusing, as some criteria in census districting (e.g., workload) is directly related to the amount and distribution of the population and thus may change significantly between the two censuses. Any deficiencies in urban districting may lead to delay, imprecision, and unfair distribution of workload, which impose more costs to the whole process.

The most important criterion in census districting is the total workload of the blocks located in a district, as it is directly related to the time required to perform censusing. However, there are other spatial and geometrical criteria that influence the districting and can provide more efficient divisions, which are introduced and quantified in the rest of this section.

\subsection{Length of the Traversed Path}

The length of the path traversed by the census officers in a district has a direct effect on the censusing time. Two districts with similar workloads may be censused in considerably different time lengths due to difference in the length of the paths taken. 
Although traversed path length is not accurately predictable before the census procedure is actually performed, it could be estimated through three factors including the area of the districts, sum of the perimeter of the district's blocks, and length of routes of the district's blocks: Assume an urban area has been divided into $n$ census districts with the initial workload $V_{0}$; And $V_{1 \mathrm{i}}, A_{\mathrm{i}}, P_{\mathrm{i}}, L_{\mathrm{i}}$ are respectively the final workload, area, perimeter, and length of the routes for the $i^{\text {th }}$ district. Then the differences between the above values with their average in the entire area are calculated:

$$
\begin{aligned}
\Delta V_{i} & =V_{1 i}-V_{0} \\
\Delta A_{i} & =A_{i}-\bar{A} \\
\Delta P_{i} & =P_{i}-\bar{P} \\
\Delta L_{i} & =L_{i}-\bar{L}
\end{aligned}
$$

These parameters are combined in a polynomial whose coefficients are estimated through a least square calculation and the information provided by a former census:

$$
\Delta V_{i}=a_{1} \Delta A_{i}+a_{2} \Delta P_{i}+a_{3} \Delta L_{i}
$$

\subsection{Compactness}

Compactness of a geometric object indicates how its shape is close to a circle. It has a significant influence on census districting procedure; because the points of a circular district are more accessible for the census officers, and thus the length of the traversed path will be smaller. Among several estimations proposed for measuring the compactness of geometrical shapes (Salazar-Aguilar et al., 2011; Svec et al., 2007; Young, 1988), here the perimeter to area test (P2A) is used (Ralphs et al., 2009):

$$
\text { compactness }=\frac{1}{4 \pi} \frac{\text { perimeter }^{2}}{\text { area }}
$$

The P2A suggests that the compactness is 1 for a circle. As the shape deviates from the circle, its compactness decreases (Table $1)$.

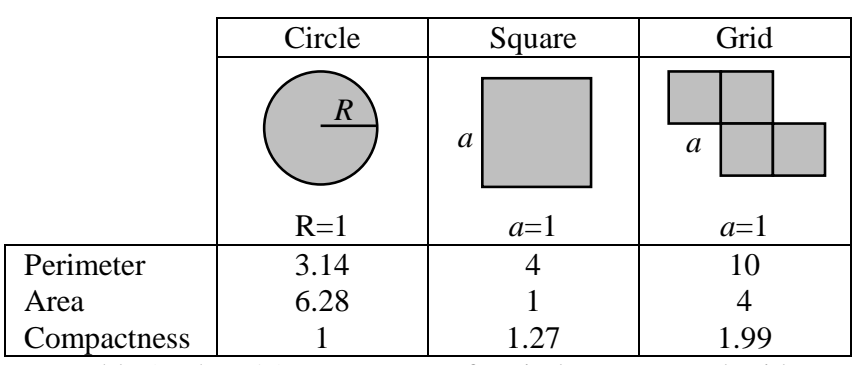

Table 1. The P2A compactness for circle, square and grid

\subsection{Natural and Artificial Barriers}

An important issue in a census districting procedure is considering the difficulties of census officers in passing some natural or artificial features (e.g., highways, rivers, etc.), which are time-consuming to pass and may affect the total path length as well as census time. In other words, lower barriers in a district will result in a faster censusing.

Here, we assign a hardness of passing value (HPV) to each barrier, which is then used to provide a measure for the barriers exist in a district. HPV is a value between 0 (for completely passable features, e.g., valleys or minor streets) and 1 (for unpassable features, e.g., rivers with no bridge). A wide avenue with pedestrian bridges has a lower HPV compare to a similar avenue with no pedestrian bridges.

\section{IMPLEMENTATION RESULTS}

The proposed approach of the paper was implemented and used to produce a census districting for Gorgan (a city in the north of Iran). The map of urban population blocks (Figure 3) was provided by the Statistical Center of Iran $^{1}$. Among other descriptive information, the number of households and the census workload are assigned to each block.

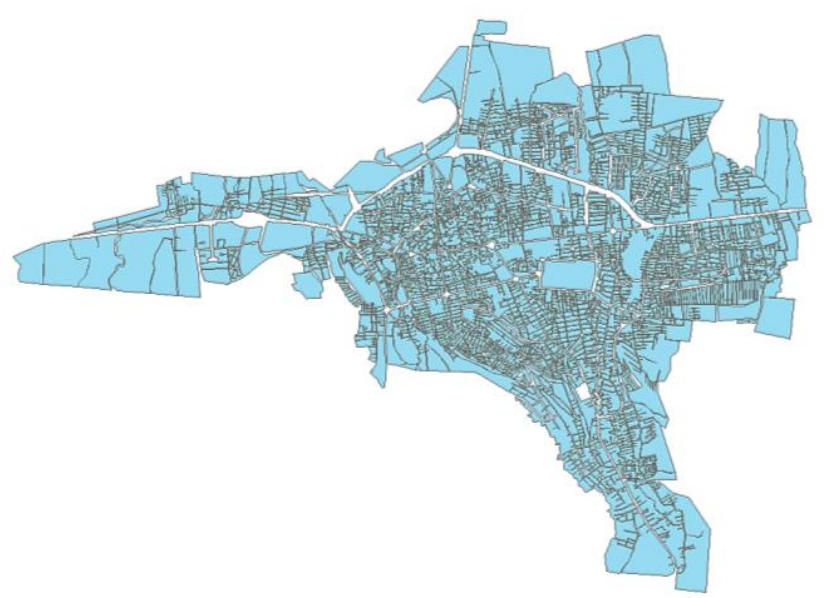

Figure 3. The map of Gorgan population blocks

An ArcGIS extension was developed that considers all of the spatial and non-spatial criteria that affect the census districting (section 3). Figure 4 illustrates the map of Gorgan's census districts produced by this extension.

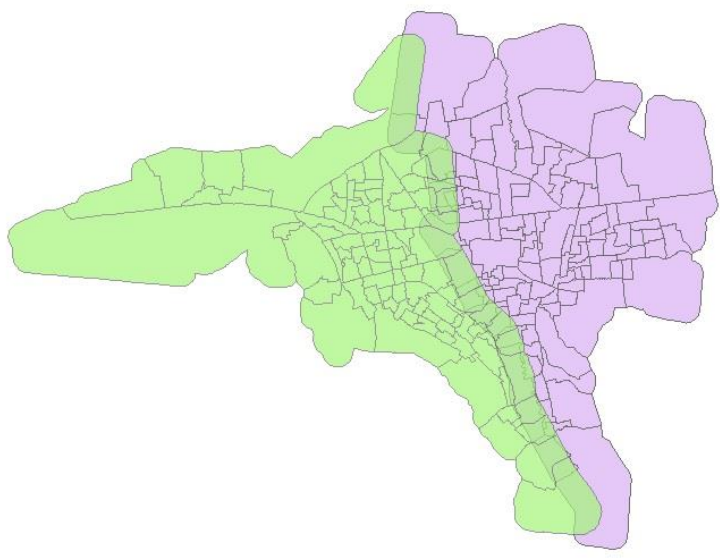

Figure 4. The map of Gorgan's census districts based on the proposed approach

In order to evaluate the efficiency of the proposed districting, the results were compared with the Gorgan's census districting that is practically used by the Statistical Center of Iran (Figure 5), which only takes the workload into account. The districts produced by the two approaches were compared in terms of 
workload, compactness and avoiding barriers, whose results are presented in the following subsections:

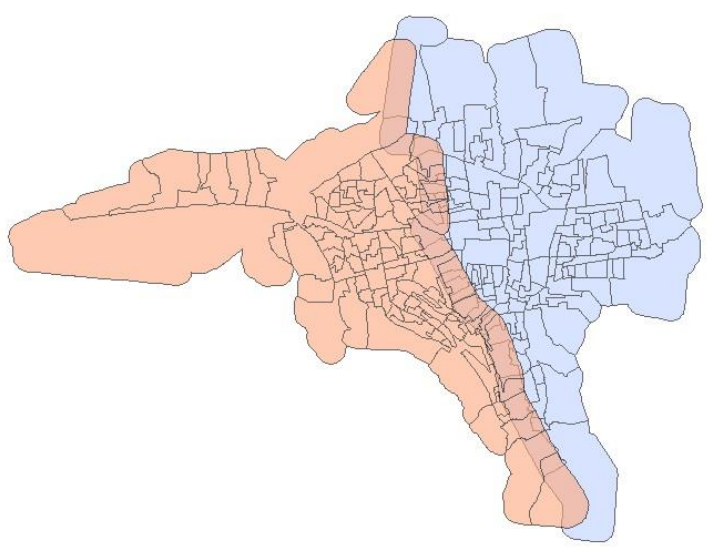

Figure 5. The map of census districts of Gorgan produced by SCI based on workloads

\subsection{Workload}

The statistical comparisons show that the workloads of the districts produced by the two approaches are fairly similar (Table 2). However, workload of the districts of the proposed approach is slightly more homogenous (compare the standard deviations in Table 2).

\begin{tabular}{|l|c|c|}
\cline { 2 - 3 } \multicolumn{1}{c|}{} & $\begin{array}{c}\text { SCI } \\
\text { approach }\end{array}$ & $\begin{array}{c}\text { Proposed } \\
\text { approach }\end{array}$ \\
\hline Number of districts & 217 & 218 \\
Average district workload & 21.01 & 20.91 \\
Standard deviation of workloads & 5.76 & 4.53 \\
Maximum district workload & 58.95 & 45.6 \\
Minimum district workload & 6.96 & 5.01 \\
\hline
\end{tabular}

Table 2. Statistical comparison of the workloads

\subsection{Compactness}

As illustrated by Table 3 and Figures 6 and 7, the proposed approach has provided more compact districts, which improves the censusing required time through reducing the length of the traversed path.

\begin{tabular}{|l|c|c|}
\cline { 2 - 3 } \multicolumn{1}{c|}{} & $\begin{array}{c}\text { SCI } \\
\text { approach }\end{array}$ & $\begin{array}{c}\text { Proposed } \\
\text { approach }\end{array}$ \\
\hline Average compactness & 2.21 & 1.83 \\
Maximum compactness & 6.42 & 3.56 \\
Minimum compactness & 1.22 & 1.19 \\
\hline
\end{tabular}

Table 3. Comparison of compactness

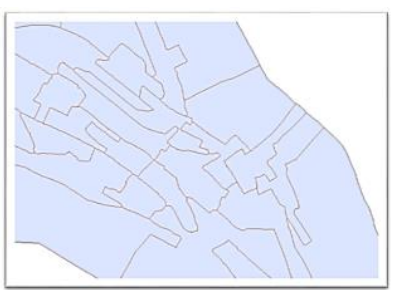

(a)

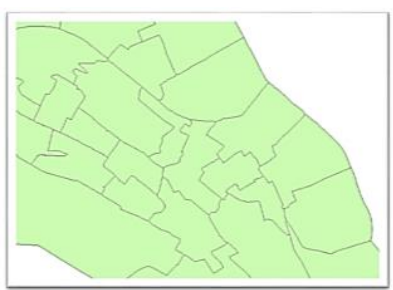

(b)
Figure 6. Comparison of the compactness for some districts produced by (a) the SCI and (b) the proposed approach

\subsection{Avoiding Barriers}

The districts produced by the two approaches were examined in terms of the number of districts that include main roads and rivers as barriers. Table 4 and Figure 8 certify the improvement achieved by the proposed approach.

\begin{tabular}{|l|c|c|}
\hline Barrier type & $\begin{array}{c}\text { SCI } \\
\text { approach }\end{array}$ & $\begin{array}{c}\text { Proposed } \\
\text { approach }\end{array}$ \\
\hline Main road & 25 & 6 \\
River & 13 & 9 \\
\hline
\end{tabular}

Table 4. Comparison of barriers

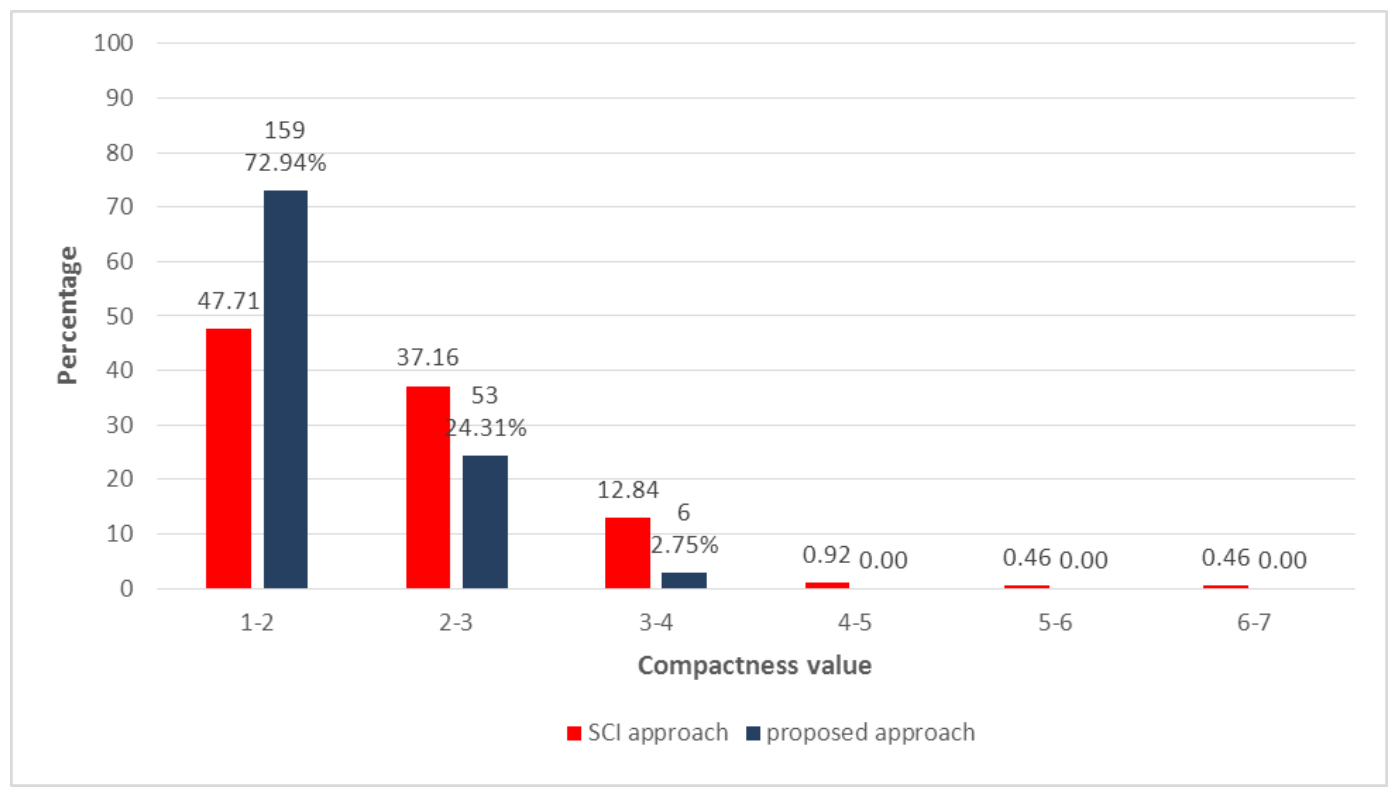

Figure 7. Distribution of the compactness values for the two approaches 


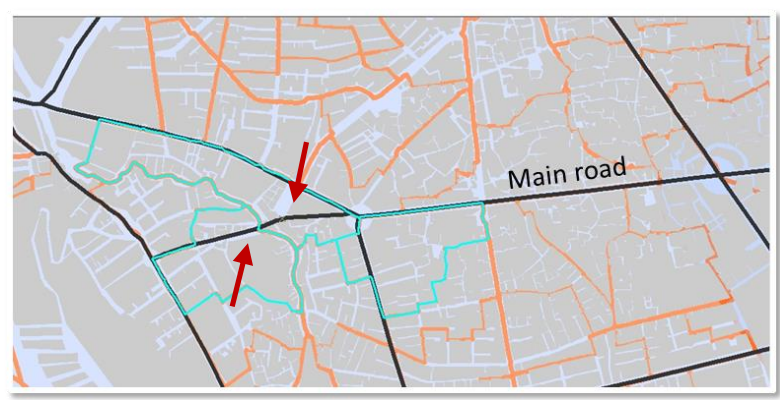

(a)

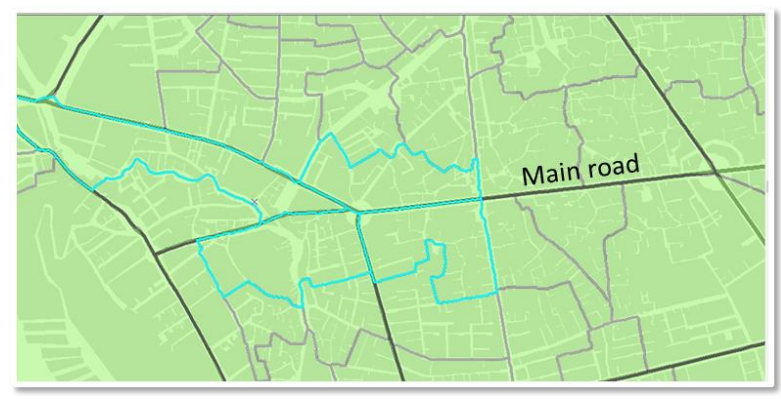

(b)

Figure 8. Comparison of the avoiding barriers for some districts produced by (a) the SCI and (b) the proposed approach. Note how the main road passes the districts produced by the SCI approach

\section{CONCLUSION AND FUTURE WORK}

This paper explores the impact of spatial aspects (e.g., traversed path length, compactness and avoiding barriers) on census districting, as an important urban districting. It proposes an approach that not only considers the workload, as the most effective criterion in census districting, but spatial criteria such as compactness, barriers and travers length are also explicitly involved. Although such criteria may be implicitly considered by the human operator while he/she is applying the census districting based on the workload, no value is provided and there is no insight on how much they are satisfied. The implementation results of the proposed approach for a case study certify that the proposed approach results in more fairly workload distribution, more compact districts and fewer number of barriers passes through the districts.

Any attempt toward improving the accuracy and speed of the census districting may underlie future research in this field. To quantify spatial criteria and generate more accurate mathematical models, useful information may be used about their effects on the workload change by qualitative and quantitative evaluation of former censusings. In addition, by comparing the predefined workloads of census districts with their practical values after completion of the census procedure, the effect of considering the spatial aspect of census districting will be studied.

The developed ArcGIS extension relies on human operators in producing census districts; it only provides the values of the criteria to the operator for the district in hand, and he/she manually tries to improve them interactively. We are working on automating or semi-automating the process in order to provide the optimum results.

\section{ACKNOWLEDGMENT}

This research was partially supported by the Statistical Center of Iran. The authors would like to deeply thank Soodabeh Zebarjad and Jafar Nazari for their contribution in providing the data and comparing the results.

\section{REFERENCES}

Alt, J.E., Frieden, J., Gilligan, M.J., Rodrik, D., Rogowski, R., 1996. The Political Economy of International Trade Enduring Puzzles and an Agenda for Inquiry. Comparative Political Studies 29, 689-717.

Bação, F., Lobo, V., Painho, M., 2005. Applying genetic algorithms to zone design. Soft Computing 9, 341-348.

Bozkaya, B., Erkut, E., Laporte, G., 2003. A tabu search heuristic and adaptive memory procedure for political districting. European Journal of Operational Research 144, 1226.

Cova, T.J., Church, R.L., 2000. Contiguity constraints for single-region site search problems. Geographical Analysis 32, 306-329.

Garfinkel, R.S., Nemhauser, G.L., 1970. Optimal political districting by implicit enumeration techniques. Management Science 16, B-495-B-508.

Helbig, R.E., Orr, P.K., Roediger, R.R., 1972. Political redistricting by computer. Communications of the ACM 15, 735-741.

Hess, S.W., Weaver, J., Siegfeldt, H., Whelan, J., Zitlau, P., 1965. Nonpartisan political redistricting by computer. Operations Research 13, 998-1006.

Macmillan, W., 2001. Redistricting in a GIS environment: An optimisation algorithm using switching-points. Journal of geographical systems 3, 167-180.

Martin, D., 1998. Optimizing census geography: the separation of collection and output geographies. International Journal of Geographical Information Science 12, 673-685.

Mehrotra, A.J., Ellis L.; Nemhauser, George L., 1998. An Optimization Based Heuristic for Political Districting. SOURCEManagement Science 44, 1100.

Moreno-Regidor, P., de Lacalle, J.G.L., Manso-Callejo, M.Á., 2012. Zone design of specific sizes using adaptive additively weighted Voronoi diagrams.

Mu, L., 2004. Polygon Characterization With the Multiplicatively Weighted Voronoi Diagram*. The Professional Geographer 56, 223-239.

Novaes, A.G.N., Souza de Cursi, J., da Silva, A.C.L., Souza, J.C., 2009. Solving continuous location-districting problems with Voronoi diagrams. Computers \& operations research 36, 40-59.

Openshaw, S., 1977. A Geographical Solution to Scale and Aggregation Problems in region-building, partitioning and spatial modelling. Transactions of the Institute of British Geographers

2, 459-472. 
Openshaw, S.a.R., L., 1995. Algorithms for reengineering 1991 Census geography. Environment and planning A 27, 425-446.

Ralphs, M., Ang, L., Zealand, S.N., 2009. Optimised geographies for data reporting: zone design tools for census output geographies. Statistics New Zealand.

Ricca, F., Scozzari, A., Simeone, B., 2008. Weighted Voronoi region algorithms for political districting. Mathematical and Computer Modelling 48, 1468-1477.

Ricca, F., Scozzari, A., Simeone, B., 2011. Political districting: from classical models to recent approaches. 4OR 9, 223-254.

Ricca, F., Simeone, B., 2008. Local search algorithms for political districting. European Journal of Operational Research 189, 1409-1426.

Salazar-Aguilar, M.A., Rıs-Mercado, R.Z., González-Velarde, J.L., 2010. A Divide-and-Conquer Approach to Commercial Territory Design.

Salazar-Aguilar, M.A., Ríos-Mercado, R.Z., González-Velarde, J.L., 2011. A bi-objective programming model for designing compact and balanced territories in commercial districting. Transportation Research Part C: Emerging Technologies 19, 885-895.

Shirabe, T., 2005. A model of contiguity for spatial unit allocation. Geographical Analysis 37, 2-16.

Shirabe, T., 2009. Districting modeling with exact contiguity constraints. Environment and planning. B, Planning \& design 36,1053 .

Svec, L., Burden, S., Dilley, A., 2007. Applying Voronoi Diagrams to the Redistricting Problem. UMAPJournal, 236.

Torpelund-Bruin, C., Lee, I., 2009. When generalized Voronoi diagrams meet GeoWeb for emergency management. Intelligence and Security Informatics, 64-75.

Wei, B.C.a.C., W.Y., 2004. A multiobjective hybrid metaheuristic approach for gis-based spatial zoning model. Journal of Mathematical Modelling and Algorithms 3, 245-261.

Yamada, T., 2009. A mini-max spanning forest approach to the political districting problem. International Journal of Systems Science 40, 471-477.

Young, H.P., 1988. Measuring the compactness of legislative districts. Legislative Studies Quarterly, 105-115.

Zoltners, A.A., Sinha, P., 1983. Sales Territory Alignment: A Review and Model. Management Science 29, 1237-1256. 